\title{
DISTRIBUIÇÃO DE FUNGOS MICORRÍZICOS NOS ASSENTAMENTOS PALMARES E ARARAS
}

\author{
Fernanda Dias Pereira ${ }^{1}$; Andrea Hentz de Mello $^{2}$; Helena de Souza Correa ${ }^{1}$; Fernando \\ Michelotti $^{3}$; Rosana Quaresma Maneschy ${ }^{3}$; Clarissa Mendes Knoechelmann ${ }^{3}$ \\ ${ }^{1}$ Discentes do curso de Agronomia, Faculdade de Ciências Agrárias de Marabá (FCAM), Universidade Federal do Pará \\ (UFPA) e bolsistas FAPESPA, fernanda_dyas@hotmail.com \\ ${ }^{2}$ Professora adjunta II, FCAM, UFPA, andreahentz@ufpa.br \\ ${ }^{3}$ Professor, FCAM, UFPA
}

\begin{abstract}
RESUMO: Os fungos micorrízicos arbusculares (FMAs) podem ser importantes para a nutrição das plantas, sobretudo em solos de baixa fertilidade e alta acidez, como é o caso dos solos da Amazônia. Diante deste contexto, este trabalho teve como objetivo à difusão da utilização de fungos micorrízicos arbusculares para a produção de mudas de essências florestais para serem empregados em sistemas agroflorestais nos assentamentos da região sudeste do Pará. O trabalho foi conduzido no período entre Março e Dezembro de 2009, através de cultivo armadilha com Brachiaria brizantha para multiplicação dos fungos e produção do inoculante a ser distribuído a partir da demanda identificada em projetos de assentamentos rurais (PAs) na região do sudeste paraense. Foram atendidas quatorze (14) famílias do P. A. Palmares e sete (7) no P. A. Araras, onde foram distribuídas um total de três mil e oitenta (3080) doses de inoculante para diversas culturas. Assim, foi verificado que existe interesse dos agricultores em melhorarem seus sistemas de cultivos através dessa tecnologia inovadora que pode corroborar para sustentabilidade dos sistemas de produção familiares.
\end{abstract}

PALAVRAS-CHAVE: Insumos biológicos, agricultura familiar, sistema de cultivo.

\section{DISTRIBUCIÓN DE HONGOS MICORRIZÓGENOS EN LOS ASENTAMIENTOS ARARAS Y PALMARES}

\begin{abstract}
RESUMEN: Hongos micorrizógenos arbusculares (HMA) pueden ser importantes para la nutrición de las plantas, especialmente en suelos de baja fertilidad y alta acidez, como es el caso de los suelos amazónicos. En este contexto, este estudio tuvo como objetivo difundir el uso de hongos formadores de micorrizas para la producción de plántulas de árboles forestales para su uso en sistemas agroforestales en los asentamientos de Pará sureste La obra se llevó a cabo entre marzo y diciembre 2009, a través de una cultura de la trampa con Brachiaria brizantha para la multiplicación de hongos y la producción del inoculo que se distribuirán de la demanda identificada en los proyectos de asentamiento en las zonas rurales (AP) en el suroeste de Pará. Se cumplieron los catorce (14) familias de P. A. Palmares y siete (7) en P. A. Araras, donde distribuyeron un total de tres mil y ochenta dosis (3080) de las diferentes culturas. Así se hizo evidente que hay interés en los agricultores a mejorar sus sistemas de cultivo a través de esta innovadora tecnología que podría ayudar a la sostenibilidad de los sistemas de producción familiar.
\end{abstract}

PALAVRAS CLAVES: Suministros biológicos, agricultura familiar, sistema de cultivo. 


\section{INTRODUÇÃO}

O Sudeste Paraense teve, nos últimos trinta anos, uma dinâmica agrária marcada por elevado crescimento populacional, intensos conflitos sociais e graves impactos ambientais. Essa situação deu-se em função do encontro de diferentes frentes de expansão que migraram para a região (VELHO, 1972), disputando o acesso às terras e recursos naturais e, sobretudo, confrontando racionalidades distintas de produção e desenvolvimento. A frente de expansão camponesa que participou desse processo chocou-se com o modelo de desenvolvimento priorizado nas décadas de 1960/70, baseado na agropecuária patronal extensiva, na concentração de terras e na simplificação do ecossistema regional com a substituição da floresta tropical por pastagens.

No entanto, face a uma ativa capacidade de mobilização política, os camponeses conseguiram se colocar como atores sociais reconhecidos no cenário regional. Maior prova disso, foram os 169 Projetos de Assentamentos conquistados entre 1987 e 2007 nos municípios que compõem o chamado Território do Sudeste Paraense. Estes ocupam uma área total de 1,2 milhões de ha e abrigam um total de 21 mil famílias de agricultores (INCRA, 2008). Além da regularização fundiária, vários recursos foram liberados na forma de crédito para a agricultura familiar, assistência técnica, projetos educacionais e de infra-estrutura.

Apesar da conquista desses benefícios durante as décadas de 1990/2000, o longo período de instabilidade fundiária anterior levou o campesinato posseiro à priorizar sistemas de produção pouco complexos, baseados sobretudo na trajetória roça-pasto (HURTIENNE, 1999). Por isso, predominaram na região trajetórias precoces de 'pecuarização' nas áreas camponesas.

Considera-se que a questão central da sustentabilidade dos sistemas de produção camponeses continuou sendo um desafio em aberto para a estabilização desses assentamentos. A experiência amazônica mostra que onde a produção familiar nas diversas formas camponesas (assentados, colonos, extrativistas, quilombolas, ribeirinhos e pescadores) conseguiu sistemas de produção melhor sucedidos foi onde ela logrou se aproximar da estrutura ecológica da floresta, ou seja, dos sistemas agroflorestais dinâmicos (SAFs).

Foi a partir disso que se tem chamado a atenção para a construção de uma identidade agroflorestal nos movimentos sociais do campo na Amazônia (GONÇALVES, 2001) com fortes benefícios econômicos (COSTA, 2000), pois os sistemas agroflorestais (SAF) constituem em alternativa econômica e ecologicamente equilibrada para a região, pois esses sistemas imitam, de certa forma, a 
floresta primária, mas com a vantagem de serem mais ricos em espécies importantes para o homem (OLIVEIRA, 1991).

Para Marques et al. (1988) os SAFs representam técnicas potenciais para 0 aproveitamento contínuo do solo, pois, ao mesmo tempo em que se produz madeira a médio e longo prazo, obtêm-se safras de cultivos agrícolas temporários ou perenes. Frente a esse quadro, uma questão emerge: como superar a dificuldade inicial de introdução de sistemas agroflorestais por camponeses assentados?

Neste contexto, têm-se buscado alternativas que racionalizem o uso sustentável dos recursos naturais dessa região, minimizando a utilização de insumos químicos, como a adoção de estratégias biológicas, com destaque para as associações micorrízicas arbusculares, por apresentarem benefícios em muitas espécies de plantas em condições de estresses, principalmente em solos de baixa fertilidade.

As associações micorrízicas propiciam melhor resistência ao estresse hídrico, temperaturas elevadas, acidez e à maior tolerância às condições de toxidez do solo e proteção do sistema radicular contra patógenos. Com isso, contribuem para o estabelecimento e desenvolvimento das plantas, mesmo em solos com baixos teores de nutrientes ou degradados (MARX;
CORDELL, 1989), como os da região amazônica.

$\mathrm{O}$ presente trabalho de pesquisa $\mathrm{e}$ desenvolvimento pretende contribuir com essa superação, desenvolvendo uma tecnologia inovadora de produção de mudas de alta qualidade de espécies arbóreas de interesse econômico para a produção familiar da região. Trata-se da criação de um banco de inoculo de fungos micorrízicos arbusculares e produção de mudas inoculadas com estes fungos com potencial de desenvolvimento de relações simbiônticas com as raízes das mudas, e mais especificamente identificar agricultores interessados em implantar esta tecnologia em seus sistemas de cultivo.

\section{MATERIAL E MÉTODOS}

Para a criação de banco de inóculo foram extraídos esporos de fungos micorrízicos arbusculares de amostras de solos coletados em áreas de cultivo do Projeto de Assentamento (P. A.) Araras, situado no Município de São João do Araguaia-PA e do P. A. Palmares no Município de ParauapebasPA realizados em um levantamento anterior que identificou a ocorrência dos gêneros Acaulospora, Gigaspora, Glomus e Scutellospora (HENTZ et al., 2009).

A identificação foi realizada a partir de coletas de amostras de solos e de raízes de plantas em áreas de plantios de açaí (Euterpe oleracea Mart.), acerola (Malpighia 
emarginata), castanha (Bertholletia excelsa), cupuaçu (Theobroma grandiflorum), côco (Cocos nucifera), laranja (Citrus x sinensis), limão (Citrus $x$ limon), pupunha (Bactris gasipaes), goiaba (Psidium guajava), cajá (Spondias lutea), caju (Anacardium orcidentale) abacaxi (Ananas comosus) e maracujá (Passiflora maliformis), espécies estas de grande importância para a agricultura familiar da região.

Com essas amostras, foi realizada a identificação dos fungos do solo $\mathrm{e}$ da colonização das raízes no Laboratório de Microbiologia da Faculdade de Ciências Agrárias a partir de métodos de identificação direta (ID), que envolveu a extração de esporos e indireta (II), que foi feito em casa de vegetação, com cultivo armadilha de Brachiaria brizantha.

Na identificação direta, foi realizada a extração de esporos pelo método de peneiramento úmido (GERDEMANN; NICHOLSON, 1963) e centrifugação em sacarose (JENKINS, 1964). O procedimento de extração de esporos de FMAs do solo, foi feito a partir de uma amostra de solo, composta de 10 sub-amostras coletadas na profundidade de $0-20 \mathrm{~cm}$. Desta amostra composta de solo, foi retirada uma amostra de $50 \mathrm{~g}$, a partir da qual foi realizada a contagem dos esporos. Em seguida, os esporos, foram preparados em lâminas e identificados segundo suas características morfológicas
(SCHENCK; PÉREZ, 1987; INVAM, 2001).

Outra parte do solo foi utilizada para identificação indireta dos FMAs. Esse método teve por objetivo a recuperação das espécies de fungos, que não estavam esporulados no momento da coleta.

As unidades experimentais foram vasos de $1000 \mathrm{~g}$ de capacidade, nos quais, foi feita a semeadura com Brachiaria brizantha, em areia estéril, utilizando-se uma alta densidade de semeadura, visando forçar o desenvolvimento do sistema radicular. Os esporos selecionados e identificados das diversas áreas e cultivos foram inoculados nesta areia e ficaram em casa de vegetação por aproximadamente 150 dias. Na avaliação da colonização micorrízica nas raízes das plantas, estas foram clarificadas e coradas, segundo a metodologia de Koske e Gemma (1989) e Grace e Stribley (1991). A porcentagem do comprimento de raízes colonizadas foi avaliada pelo método da intersecção em placa quadriculada descrito no trabalho de Giovanetti e Mosse (1980), adaptado à partir do método de medidas de comprimento de raízes de Newman (1966).

A multiplicação dos fungos extraídos foi feita em casa de vegetação da Faculdade de Ciências Agrárias de Marabá - PA, onde os gêneros de fungos identificados (Acaulospora, Gigaspora, Glomus e Scutellospora) foram multiplicados em culturas armadilhas de Brachiaria brizantha 
para a produção de inóculo. Para isso foram utilizados setenta e dois (72) vasos de cultivo armadilha, que foram regados três vezes ao dia e permaneceram no local por 150 dias.

Foram realizadas palestras para a difusão da tecnologia em assentamentos rurais onde a Universidade Federal do Pará conduz projetos de pesquisa e desenvolvimento com a temática agroflorestal e para a identificação dos agricultores interessados foram realizadas visitas as unidades familiares e entrevistas não diretivas com os agricultores, e posteriormente distribuídos as doses de inóculo.

\section{RESULTADOS E DISCUSSÃO}

A multiplicação dos fungos foi realizada de forma satisfatória, visto que houve a produção de inoculantes suficiente para atender a demanda identificada nos assentamentos, que no primeiro momento, foi de três mil e oitenta (3080) doses de inoculantes, sendo cada dose composta de 3 gramas de inoculo de fungos micorrízicos arbusculares. De acordo com a tabela 1 nota- se que no PA Araras as espécies de interesses citadas pelos agricultores para a inoculação são em sua maioria frutíferas, enquanto que no PA Palmares são espécies madeiráveis. Isso acontece por que existem vários projetos que recebem apoio da Universidade Federal do Pará (UFPA), e são diretamente vistoriados e orientados por profissionais qualificados da Faculdade de Ciências Agrárias.

A utilização dos fungos micorrizicos arbusculares veio com interesse em testar essa tecnologia inovadora em seus sistemas de cultivo, sobretudo para a implantação de SAF, pois segundo Miranda (2006), a maioria das espécies arbustivas e arbóreas tropicais, frutíferas e florestais com micorrizas, desenvolvem-se melhor e mais rapidamente, podendo ser disponibilizadas mais cedo para o produtor. Isso é justificado pelo fato de as micorrizas apresentarem uma rede de hifas extracelulares que aumentam significativamente a área de absorção das raízes, melhorando os aspectos nutricionais da planta e ampliando sua capacidade inicial de crescimento e estabilização.

Tabela 1. Distribuição de inóculos nos Assentamentos Araras e Palmares localizado no Sudeste paraense.

\begin{tabular}{c|c|c|c}
\hline Assentamento & $\begin{array}{c}\text { Número de } \\
\text { famílias atendidas }\end{array}$ & Espécies de interesse para inoculação & $\begin{array}{c}\text { Quantidade } \\
\text { de inóculos }\end{array}$ \\
\hline Araras & 10 & $\begin{array}{c}\text { Acerola (Malpighia emarginata), cupuaçu (Theobroma } \\
\text { grandiflorum), cocô (Cocos nucifera), laranja (Citrus } x \text { sinensis), } \\
\text { leucena (leucena leucocephala) e limão (Citrus } x \text { limon) }\end{array}$ & $\begin{array}{c}2000 \\
\text { Palmares }\end{array}$ \\
\hline 14 & $\begin{array}{c}\text { Açaí (Euterpe oleracea), castanha do Brasil (Bertholletia } \\
\text { excelsa), ipê roxo (Tabebuia avellanedae) } \\
\text { e ipê amarelo (Tabebuia chrysotricha) }\end{array}$ & 1080 \\
\hline
\end{tabular}




\section{CONCLUSÕES}

Houve um grande interesse dos agricultores em melhorarem seus sistemas de cultivos, e este trabalho de pesquisa e desenvolvimento possibilitou difusão de tecnologia inovadora que proporciona melhores condições de nutrição às plantas, corroborando para sustentabilidade dos sistemas de produção familiares.

\section{REFERÊNCIAS}

COSTA, F. de A. Formação Agropecuária da Amazônia: os desafios do desenvolvimento sustentável. Belém: NAEA/UFPA, 2000. 355 p.

GONÇALVES, C. W. P. Amazônia, amazônias. São Paulo: Contexto, 2000. 178 p.

GERDEMAN, J. W.; NICOLSON, T. H. Spores of mycorrhizal Endogone species extracted from soil by wet and decanting. Trans. Br. Myc. Soc. v.46, p. 235-244, 1963.

HENTZ, A. M.; NASCIMENTO, S. F.; CORRÊA, H. S.; PEREIRA F.D.; BOFF, V. F. Diversidade de Esporos de Fungos Micorrízicos Arbusculares em Ecossistemas nos Projetos de Assentamento Araras e Palmares no Sudeste Paraense, 2009. In: CONGRESSO BRASILEIRO DE CIÊNCIA DO SOLO, 32. Fortaleza. Anais... Fortaleza: SBCS, 2009. CD-ROM.

HURTIENNE, T. Agricultura Familiar na Amazônia Oriental: uma comparação dos resultados da pesquisa sócio-econômica sobre fronteiras agrárias sob condições históricas e agro-ecológicas diversas. Novos Cadernos NAEA, v. 2, n. 1, p. 75-94, 2000.

INSTITUTO NACIONAL DE COLONIZAÇÃO E REFORMA AGRÁRIA INCRA. Sistema de informações sobre projetos de reforma agrária -SIPRA. [S.1: s.n]. 2007.
INTERNATIONAL

CULTURE COLLECTION

$\mathrm{OF}$ VESICULAR ARBUSCULAR MYCORRHIZAL FUNGI INVAM, 2001. Disponível em: $<$ http://invam.caf.wvu.edu/mycinfo/methods/cult ures/monosp.htm>. Acesso em: março de 2007.

JENKINS, W. R. Arapid centrifugal-floration technique for separating nematodes from soil. Plant Disease Report, v.48, p. 692,1964.

MARX, D. H.; CORDELL, C. E. The use of specific in ectomycorrhizas to improve artificial forestation practices. In: WHIPPS, J. M.; LUMSDEN, R. D. (Ed.). Biotechnology of fungi for improving plant growth. New York: Academic Press, p. 1-25, 1989.

MIRANDA, J. C. C. Porque a micorriza é importante para a produção agrícola, frutífera e florestal, 2006. Disponível em: < http://www.agrosoft.org.br/agropag/20721.ht m >. Acesso em: 14 mai. 2010.

OLIVEIRA, L. A.; MOREIRA, F. M. S.; MOREIRA, F. W. Ocorrências de microrganismos benéficos e ecossistemas amazônicos. In: NODA, H.; SOUZA, L. A. G.; FONSECA, O. J. M. (Eds.) Duas décadas de contribuições do INPA à pesquisa agronômica no trópico úmido. Manaus: INPA, 1997. p. 221-240.

SCHENCK, N.; PERÉZ, Y. Manual for the identification of VA mycorrhizal fungi. 3.ed. Gainesville: Synbergistic Publications, 1987. 286 p.

VELHO, O. G. Frentes de expansão e estrutura agrária: Estudo do processo de penetração numa área da Transamazônica. Rio de Janeiro: Editora Zahar, 1978. 19 p.

\section{AGRADECIMENTOS}

O presente trabalho foi realizado com o apoio da Fundação de Amparo a Pesquisa do Estado do Pará - FAPESPA. 